\title{
Discovery of global genomic re-organization based on comparison of two newly sequenced rice mitochondrial genomes with cytoplasmic male sterility-related genes
}

\author{
Sota Fujii ${ }^{1,2}$, Tomohiko Kazama $^{1}$, Mari Yamada', Kinya Toriyama ${ }^{1 *}$
}

\begin{abstract}
Background: Plant mitochondrial genomes are known for their complexity, and there is abundant evidence demonstrating that this organelle is important for plant sexual reproduction. Cytoplasmic male sterility (CMS) is a phenomenon caused by incompatibility between the nucleus and mitochondria that has been discovered in various plant species. As the exact sequence of steps leading to CMS has not yet been revealed, efforts should be made to elucidate the factors underlying the mechanism of this important trait for crop breeding.

Results: Two CMS mitochondrial genomes, LD-CMS, derived from Oryza sativa L. ssp. indica (434,735 bp), and CW-CMS, derived from Oryza rufipogon Griff. (559,045 bp), were newly sequenced in this study. Compared to the previously sequenced Nipponbare (Oryza sativa L. ssp. japonica) mitochondrial genome, the presence of 54 out of 56 protein-encoding genes (including pseudo-genes), 22 tRNA genes (including pseudo-tRNAs), and three rRNA genes was conserved. Two other genes were not present in the CW-CMS mitochondrial genome, and one of them was present as part of the newly identified chimeric ORF, CW-orf307. At least 12 genomic recombination events were predicted between the LD-CMS mitochondrial genome and Nipponbare, and 15 between the CW-CMS genome and Nipponbare, and novel genetic structures were formed by these genomic rearrangements in the two CMS lines. At least one of the genomic rearrangements was completely unique to each CMS line and not present in 69 rice cultivars or 9 accessions of $O$. rufipogon.

Conclusion: Our results demonstrate novel mitochondrial genomic rearrangements that are unique in CMS cytoplasm, and one of the genes that is unique in the CW mitochondrial genome, CW-orf307, appeared to be the candidate most likely responsible for the CW-CMS event. Genomic rearrangements were dynamic in the CMS lines in comparison with those of rice cultivars, suggesting that 'death' and possible 'birth' processes of the CMS genes occurred during the breeding history of rice.
\end{abstract}

\section{Background}

In contrast to the compact structure of the human mitochondrial genome (13 genes in approximately 17 $\mathrm{kb})$, higher plant mitochondria are extremely complex in their constitution, and their process of evolution is enigmatic. In addition, although animal mitochondrial genomes are relatively conserved in size, from Homo sapiens to Mus musculus, the genomic content of plant

\footnotetext{
* Correspondence: torikin@bios.tohoku.ac.jp

'Laboratory of Environmental Biotechnology, Graduate School of Agricultural Science, Tohoku University, 1-1 Tsutumidori-Amamiyamachi, Aoba-ku, Sendai 981-8555, Japan
}

(c) 2010 Fujii et al; licensee BioMed Central Ltd. This is an Open Access article distributed under the terms of the Creative Commons Attribution License (http://creativecommons.org/licenses/by/2.0), which permits unrestricted use, distribution, and reproduction in any medium, provided the original work is properly cited. mitochondria is highly divergent, ranging from an estimated 200 to $2,400 \mathrm{~kb}$ [1]. The conservation of simple circular mitochondrial DNA that is seen in animals is even in doubt in plants, and a number of studies have predicted that the genetic information of plant mitoaddition to the 13 housekeeping genes also found in animals, plants contain numerous open reading flames (ORFs) with unknown functions as well as genes with known functions, such as ribosomal protein subunitencoding genes, that are not observed in the animal genomes. Although most of these ORFs are considered chondria is partitioned into subgenomes [2]. Finally, in 
to be non-functional and suppressed during development, some studies have reported that several ORFs are transcribed [3]. These mysteries of the mitochondria are certainly intriguing in view of the co-evolution of nuclear and mitochondrial genomes. To date, seven mitochondrial genomes have been sequenced from plant species: four from dicot species, Arabidopsis thaliana [4], Beta vulgaris [3,5], Brassica napus [6], and Nicotiana tabacum [7], and three from monocot species, Oryza sativa [8,9], Zea mays $[10,11]$, and Triticum aestivum [12]. These studies have shown that plant mitochondrial genomes are actually divergent in their noncoding sequences and even in their gene-coding regions; for instance, the presence of ribosomal protein subunitencoding genes is known to be quite inconsistent among species $[2,13]$.

Cytoplasmic male sterility (CMS) is a maternally inherited male sterile trait, and a significant amount of evidence indicates that this phenomenon stems from alteration of the mitochondrial genome [14,15]. In many cases, the product of the unique gene structure in the CMS mitochondrial genome, consisting of a chimeric structure of endogenous gene fragments and an alien sequence, has been demonstrated to be associated with the mitochondrial inner membrane [16-19]. Most of these mitochondrial CMS-associated genes (MCAG) are thought to form a pore within the membrane and, although the details of the mechanism from pore-forming to CMS occurrence have not been fully elucidated, it is speculated that MCAGs are responsible for CMS. Since CMS is often associated with cytoplasmic substitutions, such as successive backcrossing of distantly related strains, CMS is the key phenomenon that may shed some light on plant mitochondrial-nuclear interactions.

Studies of the CMS mitochondrial genomes in B. vulgaris [3] and Z. mays [11] revealed the presence of several large recombination events compared to normal cytoplasm. Satoh et al. [3] sequenced the Owen-CMS mitochondrial genome in sugar beet, and a comparison with the non-CMS mitochondria revealed the existence of several CMS-specific ORFs generated by the genomic rearrangements. PreSatp6 was later determined to be the best candidate for MCAG, and the gene products of PreSatp6 were localized in the mitochondrial inner membrane in the form of a homo-oligomer [18]. Allen et al. [11] sequenced five distinct mitochondrial genomes in maize and detected massive genomic recombinations among the lines. Even two of the normal cytoplasms which were sequenced, NA and NB, were significantly dissimilar to each other, and it was estimated that there were 16 genomic rearrangements between the lines. They reported that genome sizes of the lines ranged from 535,825 bp in CMS-T to 739,719 bp in CMS-C [11].
The 490,520-bp mitochondrial genome of rice (Oryza sativa L.) japonica cultivar Nipponbare was sequenced, and 53 protein-encoding genes, 17 tRNA genes, and 3 rRNA genes were predicted [8]. When the existence of the master circle was assumed, at least three large duplications $(>10,000 \mathrm{bp})$ were found, and portions of plastid and nuclear sequences were frequently inserted, making the genome even more complex. The mitochondrial genome of the indica strain 93-11 was also assembled, and it was predicted to possess 491,515-bp nucleotides [9]. At the same time, the 490,673-bp mitochondrial genome was assembled for the strain PA64S carrying japonica cytoplasm. A total of 96 SNPs and 25 Indels were predicted between 93-11 and PA64S, but the differences in their overall genomic structures were rather conservative, in comparison to the report that the sequence arrangements of two normal cytoplasms from maize were never in parallel [11].

Based on this background, our intention was to determine whether the characteristics of CMS mitochondrial sequences of rice are comparable with those of maize or sugar beet. In rice, more than 60 types of CMS have been reported (see [20] or [21] for reviews); however, the mitochondrial sequence has not yet been revealed for any CMS rice. In this study, we employed two independent CMS lines, LD-CMS and CW-CMS, derived from the Burmese strain Lead rice (Oryza sativa L. ssp. indica) and from the Chinese wild rice strain W1 (Oryza rufipogon Griff.), respectively. Using the Nipponbare mitochondrial sequence as a reference, we chose the high-throughput pyrosequencing method to gather information about the rice CMS mitochondrial genomes. A number of genomic rearrangement events were detected between the two CMS mitochondria in comparison with that of Nipponbare.

\section{Results}

Sequence assemblies and assessment of genomic arrangements in CW-CMS and LD-CMS mitochondrial genomes

The CW-CMS mitochondrial genome was assembled to 559,045-bp single circular sequences, whereas the LDCMS mitochondrial genome was predicted to have 434,735 bp when the master circle was hypothesized. When compared with the previously assembled Nipponbare (490,669 bp), PA64S (490,673 bp), and 93-11 (491,515 bp) [9] genomes, the sequence length of the two CMS lines differed greatly (Table 1 ). Thus, we speculated that the predicted mitochondrial genomic organization of the CMS lines is quite inconsistent with that of the known cultivars. We plotted the syntenic regions via the bl2seq algorithm using the Nipponbare sequence, excluding three large $(>10,000 \mathrm{bp})$ duplicated regions (Figure 1). When the entire Nipponbare 
Table 1 Summary of genomic organization.

\begin{tabular}{cccccc}
\hline & Nipponbare & PA64S & 93-11 & CW-CMS & LD-CMS \\
Accession no. & DQ167400 & DQ167807 & DQ167399 & AP011076 & AP011077 \\
\hline Origin of cytoplasm & japonica & japonica & indica & wild-rice & indica \\
Length & 490,669 & 490,673 & 491,515 & 559,045 & 434,735 \\
Synonymous mutations* & - & 0 & $3^{* * *}$ & 1 & 0 \\
Non-synonymous mutations* & - & 0 & 3 & 5 & 1 \\
Large duplications (>10,000 bp)** & 3 & 3 & 0 & 15 & 12 \\
Recombination (vs Nipponbare) & - & 0 & 0 & 1 & 2 \\
Large InDels (>1,000 bp) & - & 0 & & & 5 \\
\hline
\end{tabular}

*In comparison with Nipponbare within genic regions.

** In comparison with Nipponbare (w/o dup.)

*** Referred in [9]

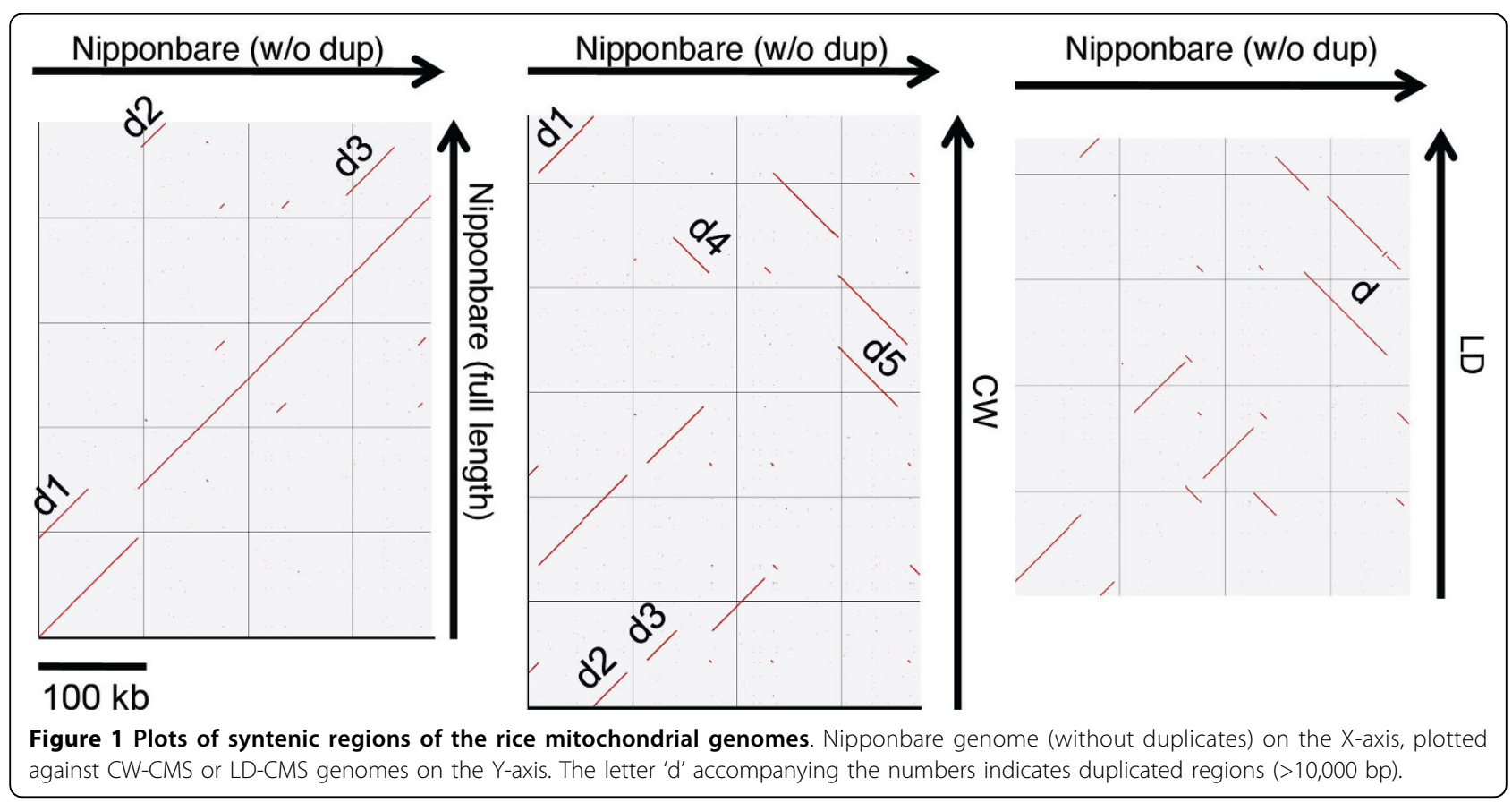

sequence including duplicated regions (Nipponbare fulllength) was plotted against its truncated version (Nipponbare w/o dup.), we detected the presence of three large redundant regions in the same positions where we deleted duplications. However, when we plotted the sequences from the two CMS lines to the same reference genome (Nipponbare w/o dup.), we found that the syntenic regions were largely discrepant in their genomic positions (Figure 1). In comparison with Nipponbare, which has three large duplications ( $>10,000 \mathrm{bp}$ ), we predicted five duplications in the CW-CMS genome and one in the LD-CMS genome (Table 1, Figure 1). This feature is consistent with the observation in maize mitochondrial comparative sequence analysis that the large duplications accounted for most of the genome size increases in mitochondria [11], and the largest genome (CW-CMS: 559,045 bp) outnumbered the smallest genome (LD-CMS: 434,735 bp) in large duplication events. The number of recombination events was at least 15-fold higher in the CW-CMS genome compared to Nipponbare and at least 12-fold higher in the LD-CMS genome. The hypothetical master circle of CMS mitochondrial genomes is displayed in Figure 2.

\section{Comparison of nucleotide sequences in genic regions}

We compared nucleotide sequences among five mitochondrial genomes: Nipponbare, PA64S, 93-11, and the two CMS lines. First, 56 protein-encoding genes (including pseudo-genes), 22 tRNA genes (including pseudotRNA), and three rRNA genes found in Nipponbare were searched in the other genomes (list in Additional file 1, Table 2, Table 3). Including genes of unknown functions and ribosomal protein subunits, the appearances of the majority of the genes were constant among 

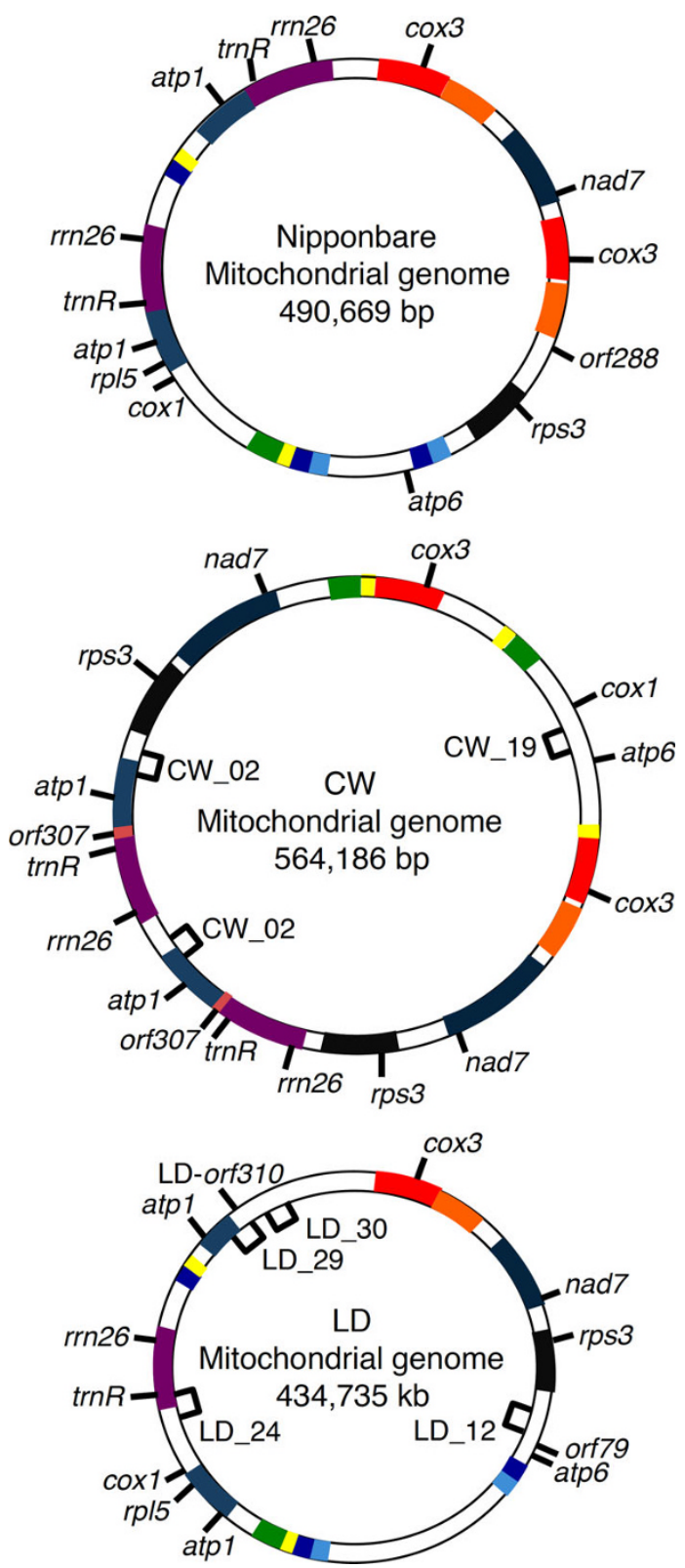

Figure 2 Hypothetical master circles of the CW and LD mitochondrial genomes, and that of Nipponbare as a reference [8]. Homologous regions are indicated by boxes of the same colors. Approximate regions amplified by the primers used in Additional file 6 are also indicated with brackets.

the five genomes. An exception was found for two ORFs which were missing from the CW-CMS genome. Due to the N-terminal deletion, the orf $152 b$ found in Nipponbare was shortened to an ORF of 92 amino acids in the CW-CMS genome (Table 3, Figure 3). Only the $\mathrm{N}$-terminus $294 \mathrm{bp}$ sequences of orf288 were present in CW-CMS (CW-orf307), whereas the orf288-like sequence was present but elongated to an ORF encoding
310 amino acids with a recombination event at the sequence corresponding to its $\mathrm{N}$-terminal region in the LD-CMS genome (LD-orf310, Figure 3).

We found four non-synonymous mutations in the CW-CMS genome (Table 2). Two point mutations caused a single amino acid change from arginine to glycine (R144G) in rps1 in the CW-CMS genome (Table 2). For rps2, two point mutations caused two amino acid changes, K183N and F184Y (Table 2). On the other hand, no amino acid changes were detected in the protein encoded by the LD-CMS genome in comparison to that of Nipponbare (Table 2), other than the 6-bp deletion in the pseudorps14 gene-like structure similarly observed in CW. The presence and the sequences of the electron chain subunitencoding genes, $n a d, c o b, c o x$, and $a t p$, were completely conserved among the CMS lines and Nipponbare.

\section{Evaluation of known SNPs in 93-11 vs PA64S}

As the genomic arrangements of the CMS lines and the previously sequenced cultivars were quite divergent (Figure 1), alignment of the whole genomes to detect SNPs or InDels was difficult. Thus, we decided to analyze 96 SNPs, 25 InDels, and three segmental sequence variations (SSV) detected by comparing 93-11 and PA64S in the study by Tian et al. [9]. The genotypes in the sequence variations are summarized in Additional files 2 and 3. Of the non-redundant 70 SNPs, only 59 SNPs were detectable in the CW-CMS and LD-CMS genomes, due to genomic deletions (Additional file 2). All of the SNP genotypes in the CW-CMS and LD-CMS genomes were the same (Additional file 2). Approximately $57 \%$ of the SNP genotypes were the same as those shared by the two japonica genomes (designated j); $14.3 \%$ were shared with the indica strain 93-11, 8.3\% with PA64S (p), 2.9\% with SNPs shared by $93-11$ and Nipponbare $(9 / \mathrm{n})$, and $1.4 \%$ were unique to the two CMS genomes.

Non-redundant InDels and SSVs were also surveyed and, in parallel to the SNPs, the genotypes of the two CMS lines were completely identical (Additional file 3). Approximately $81 \%$ of the genotypes in the CMS lines were equal to japonica type, whereas $14.8 \%$ were equal to 93-11 type. One InDel was highly variable, and we were able to distinguish all lines in this region except for the CMS lines (InDel 200488, Additional file 3). From the above results, the CMS genomes were rather close to japonica genomes when only the nucleotide sequences were taken into consideration. Overall, the sequence variations that we could detect between the two CMS lines encompassed: the elongation of orf 288 into orf310 in the LD-CMS genome, whereas orf 288 was lost from the CW-CMS genome; the absence of orf $152 b$ from the CW-CMS genome; and mutations in rps1 and rps2 (Figure 3, Additional file 2). Direct sequence comparison of the syntenic regions of the CW-CMS and 
Table 2 Summary of SNPs in genic regions in comparison with Nipponbare.

\begin{tabular}{|c|c|c|c|c|c|c|c|c|}
\hline \multirow[b]{2}{*}{ Genes } & \multicolumn{4}{|c|}{ Numbers of synonymous mutations } & \multicolumn{4}{|c|}{ Numbers of non-synonymous mutations } \\
\hline & CW & LD & PA64S & 93-11 & CW & LD & PA64S & $93-11$ \\
\hline $\cos 3$ & - & - & - & 2 & - & - & - & $1(\mathrm{~K} 3 \mathrm{Q})^{*}$ \\
\hline orf224 & 1 & - & - & 1 & $1(\mathrm{G} 3 \mathrm{~N})$ & - & - & $1(\mathrm{~S} 186 \mathrm{~L})^{*}$ \\
\hline rps1 & - & - & - & - & $2^{* *}(\mathrm{R} 144 \mathrm{G})$ & - & - & - \\
\hline rps2 & - & - & - & - & 2 (K183N, F184Y) & - & - & - \\
\hline
\end{tabular}

* Two nucleotide mutations resulted in one amino acid exchange.

Table 3 Summary of InDels and other mutations in genic regions in comparison with Nipponbare.

\begin{tabular}{|c|c|c|c|c|c|c|c|c|}
\hline \multirow[b]{2}{*}{ Genes } & \multicolumn{4}{|c|}{ Numbers of deletions } & \multicolumn{4}{|c|}{ Other mutations } \\
\hline & CW & LD & PA64S & $93-11$ & CW & LD & PA64S & $93-11$ \\
\hline $\cos 3$ & - & - & - & - & - & - & - & - \\
\hline orf152b & - & - & - & - & $\mathrm{N}$-terminal deletion & - & - & - \\
\hline orf176 & - & - & - & 9 & - & - & - & - \\
\hline orf288 & - & - & - & - & Partially present & $\operatorname{orf310*}(\mathrm{L} 162 \mathrm{P})$ & - & - \\
\hline pseudo-rps 14 & 6 & 6 & - & - & - & - & - & - \\
\hline
\end{tabular}

* ORF elongation by nucleotide changes in the N-terminal region. L162P is the amino acid change in orf288 basis.

LD-CMS mitochondrial genomes revealed 43 SNPs and InDels in total. Comparison of the syntenic regions of the CW-CMS and Nipponbare genome revealed 206 SNPs and IndDels, although it is possible in this case that some of these differences may result from the different sequencing technologies employed. Thus the two CMS genomes were relatively similar to one another in comparison to the other mitochondrial genomes. The phylogenetic relationship estimated from all SNPs of both genic and non-genic regions is displayed as a dendrogram in Additional file 4.

\section{Gene structures unique in CMS lines}

Similar to the studies that reported CMS-specific gene structures in the CMS mitochondrial genome of other species $[3,11]$, we also found DNA rearrangements generating several CMS-specific structures. We previously identified a B-atp6-orf79-like structure in the LD-CMS mitochondrial genome, L-atp6-orf79 [22]. orf79 is the strongest MCAG candidate in BT-CMS rice [23-26]. Pyrosequencing enabled us to analyze the surrounding region of L-atp6-orf79 (Figure 3C). L-atp6-orf79 was on the long syntenic atp 6 region with Nipponbare, but 6,881-bp alien sequences were inserted immediately after atp6 (Figure 3C). The insertion of 6,881 bp resulted in the generation of orf79. We have yet to determine the origin of the insertion sequences, but at least we were able to determine that the sequence was not present in Nipponbare, PA64S, 93-11, or the CWCMS line. The L-atp6-orf79 transcripts are co-transcribed in the CMS line and the inter-genic region of the transcripts is cleaved by the action of the fertility restorer gene $R f 1$ [22]. The precise expression analysis of this L-atp6-orf79 locus was conducted in our previous study [22].

CW-CMS-specific genomic structures were found in the region around the $r p l 5$ locus with exclusive genomic recombination involving the orf 288 locus (Figure 4A). In Nipponbare, rpl5 was located upstream of atp1, $8.9 \mathrm{~kb}$ apart from it. In contrast, in the CW-CMS genome, the promoter region of rpl5 was substituted with other genomic regions by homologous recombination (Figure 4A). This recombination resulted in the insertion of nucleotides for 75 amino acids at the $\mathrm{N}$-terminus of rps 2 , but the insertion was not considered to affect the rpl5 ORF structure because of the stop codon between partial rps 2 and $r p l 5$ (Figure 4B). Full-length rps 2 was found in another location in the CW-CMS genome (data not shown).

A rather interesting finding was the fate of the region around the atp 1 locus because it was involved in the generation of the chimeric ORF gene, which was comprised of multiple recombination events (Figure 4A). The upstream region of atp 1 was fused to a 294-bp sequence of orf 288 corresponding to its $\mathrm{N}$-terminal region (Figure 4A), and it was further followed by 1,369-bp unknown sequences and finally linked to the $t r n R$ region which is $26.0 \mathrm{~kb}$ away from atp 1 in Nipponbare (Figure 4A). The 1,369-bp unknown sequence found in this locus was not found in the Nipponbare, PA64S, 93-11, or LD-CMS genomes, although some of its fragments showed $85 \%$ similarity to orf224 (Figure 5). As we stated previously, a part of orf 288 was present in this region but the full-length copy was not present. The newly identified gene in this locus, possibly encoding a protein of 307 amino acids, was designated as 


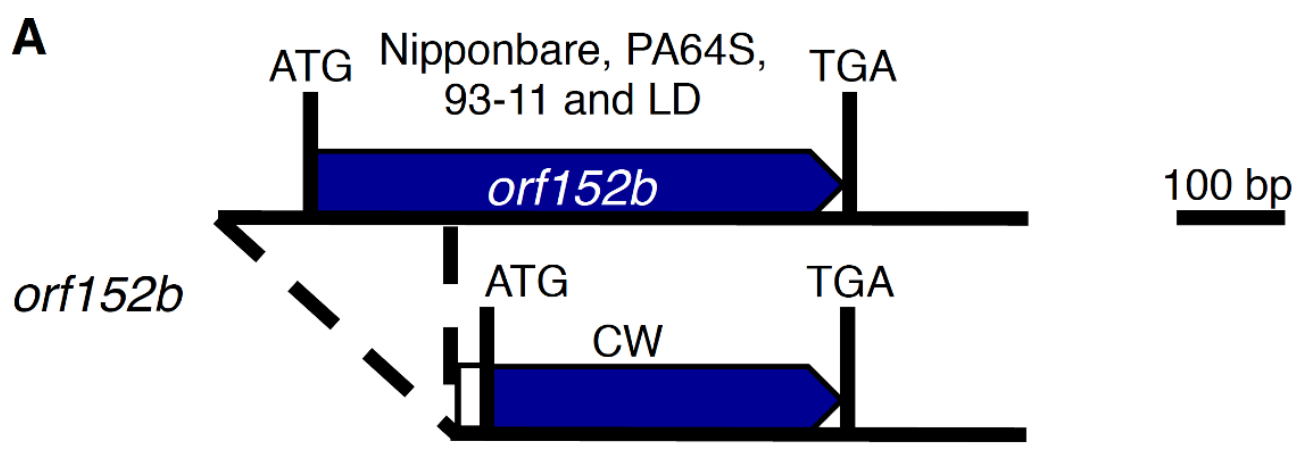

B<smiles>[B]</smiles>

L-orf310

ATG

Nucleotide changes

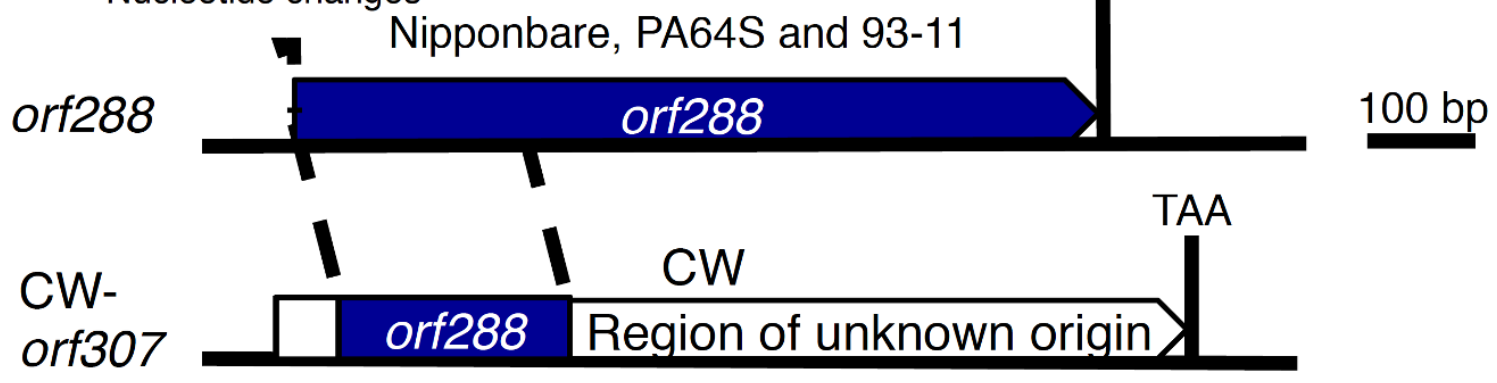

C

Nipponbare

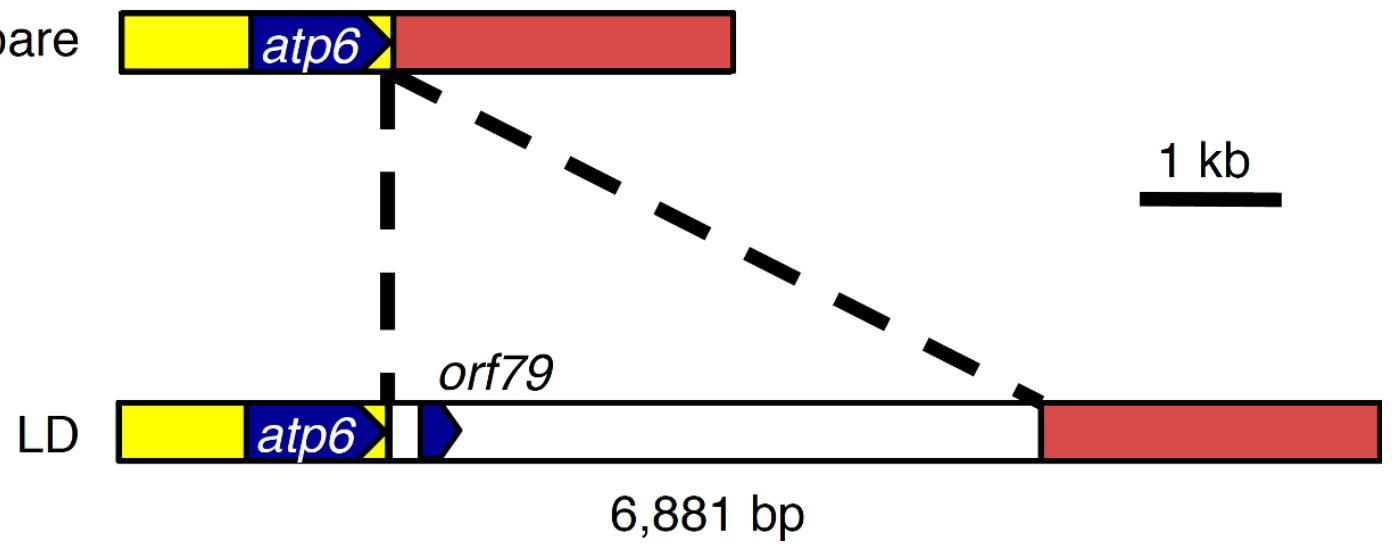

Figure 3 Major changes in gene coding regions in the CMS lines. (A) Structure of orf152b. The deletion of nucleotides in the N-terminal region shortened the ORF to a length of 92 amino acids in the CW-CMS genome. (B) Structures of orf288. Nucleotide extension at the $\mathrm{N}$-terminal region seen in the LD-CMS genome caused the generation of a novel ORF predicted to encode a 310-amino-acid polypeptide (L-orf310). The chimeric structure of partial orf288 for N-terminal peptides and sequences of unknown origin generated the CW-orf307 gene in the CW-CMS genome. (C) The 6,881-bp alien sequence insertion in the downstream region of atp6 in the LD-CMS genome. 


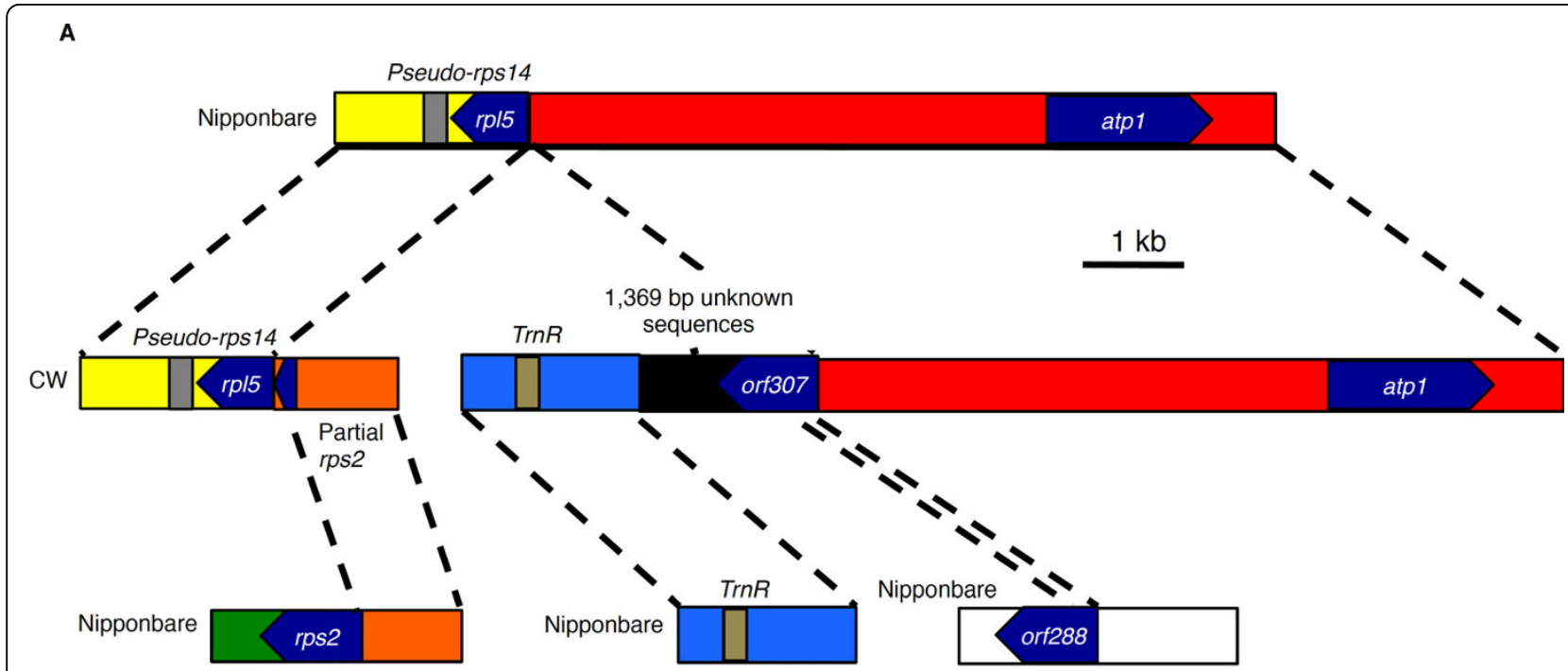

B

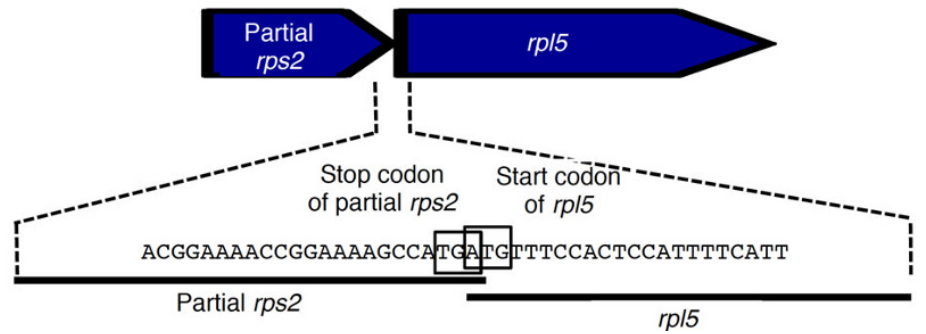

Figure 4 Genomic structures around CW-orf307 and rpl5. (A) Large-scale genomic re-organization presumed to explain the evolution of the CW-orf307 region. Boxes of same colors indicate identical regions. (B) Schematic representation of the rpl5 locus in the CW-CMS mitochondrial genome. The A nucleotide in the TGA stop codon of partial rps 2 inserted in the upstream region of rp/5 was equal to the A in the ATG start codon of rp/5. Thus, partial rps2 and rp/5 were not chimeric.

CW-orf307. The structures of the rpl5 and CW-orf307 loci identified by pyrosequencing were verified by sequencing the lambda clones covering these regions (data not shown).

\section{Genomic structures unique in CMS lines}

In order to evaluate the uniqueness of these genomic structures, distribution of the LD- and CW-specific structures and genes was surveyed in the National Institute of Agrobiological Sciences (NIAS, Japan) global rice core collection consisting of 69 accessions of worldwide cultivars [27]. This rice collection retains $90 \%$ of RFLP alleles detected in about 300 accessions selected based on the passport data from the whole rice collection (about 30,000 accessions) maintained at the NIAS Genebank [28]. Nine wild rice strains (O. rufipogon), which had previously been reported to carry a CMS cytoplasm [29], were also included in this study, so that 78 accessions were tested in total. Six primers able to amplify the contig linkage regions (described precisely in the Methods) unique in the two CMS lines in comparison with Nipponbare were used to detect each amplicon by PCR. Table 4 summarizes the results of PCR amplification and accessions were categorized into 12 mitochondrial haplogroups (A to L). No amplicon was observed in any of the accessions using primer pairs CW_02 or LD_24 (Table 4). Thus, the genomic rearrangement detected by primer pair CW_02, which corresponds to the region between the atp 1 and $r r n 26$ or rps 3 genes in CW-CMS (Figure 2), and the rearrangement detected by the primer pair LD_24, which corresponds to the region between cox 1 and $\operatorname{trnR}$ in LD-CMS (Figure 2), were completely unique to each CMS line. The range of the proportion of the accessions with amplification by four other primer pairs (CW_19, LD_12, LD_29, LD_30) was $3.8-21.8 \%$. Similar to the genomic structure detected by primer pair CW_02, CW-orf307 was solely present in the CW-CMS line (Table 4). Excluding two O. rufipogon 


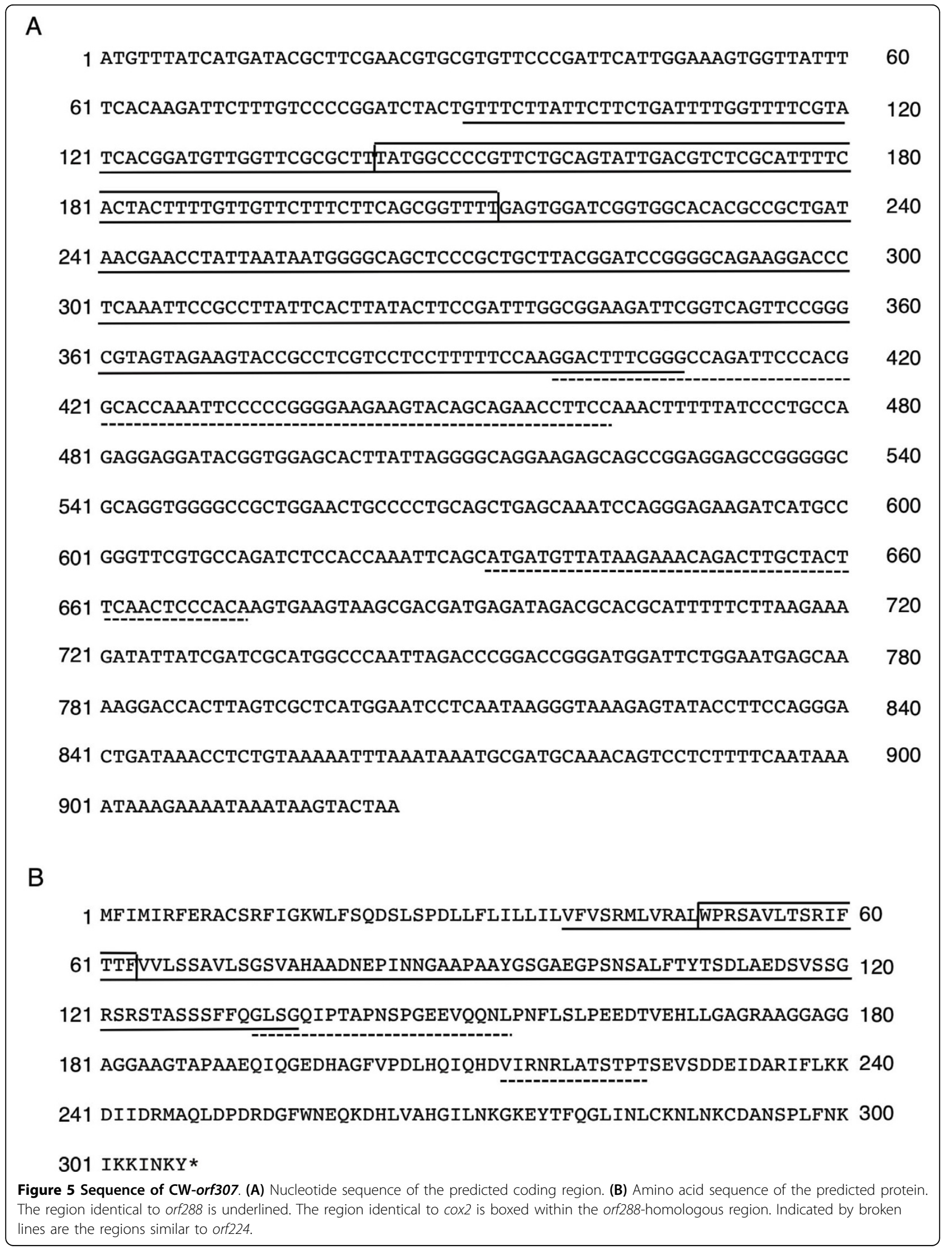


Table 4 Summary of haplogroups distinguished by PCR amplification

\begin{tabular}{|c|c|c|c|c|c|c|c|c|c|}
\hline \multirow[b]{2}{*}{ Haplogroups** } & \multicolumn{6}{|c|}{ Contig linkage primer pairs* } & \multicolumn{3}{|c|}{ Genes } \\
\hline & CW_02 & CW_19 & LD_12 & LD_24 & LD_29 & LD_30 & CW-orf307 & atp6-orf79 & orf288 \\
\hline A & - & - & - & - & - & - & - & - & + \\
\hline B & - & - & - & - & - & - & - & - & - \\
\hline C & - & + & - & - & - & - & - & - & - \\
\hline $\mathrm{D}$ & - & - & + & - & + & + & - & + & - \\
\hline E & - & - & - & - & + & + & - & - & - \\
\hline F & - & + & - & - & - & - & - & - & + \\
\hline G & - & - & - & - & - & + & - & - & + \\
\hline $\mathrm{H}$ & - & - & + & - & - & + & - & + & - \\
\hline 1 & - & - & - & - & + & + & - & - & + \\
\hline$J$ & - & - & + & - & + & - & - & + & - \\
\hline K & - & + & - & - & + & + & - & - & + \\
\hline L & - & - & - & - & + & + & - & + & - \\
\hline$C W$ & + & + & - & - & - & - & + & - & - \\
\hline LD & - & - & + & + & + & + & - & + & + \\
\hline
\end{tabular}

*See Additional File 8 for details of the primers

**Full details of haplogroups are present in Additional file 5.

accessions (W1086 and W1092, Additional file 5), the presence of the atp6-orf79 structure was completely linked with the amplification pattern of LD_12, as expected from the closeness of the two regions (Table 4). Lastly, distribution of the orf288-like gene in rice accessions was assessed, as the gene was not present in the CW-CMS genome (Figure 3, Table 4). Thirty-five accessions carried the orf288-like structure, whereas no amplification was observed in the other lines.

\section{Expression analysis of genes involved in the recombination events}

We investigated whether the genes involved in the recombination to generate the chimeric CW-orf307 locus were affected in terms of their expression patterns. As a result, $\operatorname{cox} 1, \operatorname{orf} 284$, orf165, atp 1, $\operatorname{cox} 2, \operatorname{trn} R, \operatorname{rpl5}$, and rps 2 were expressed in the CW-CMS calli (Additional file $6)$, whereas the orf224 transcripts were extensively reduced in the CW-CMS line (Additional file 6). Due to the recombination event with $r p s 2$, rpl5 probably acquired a new promoter and, as a result, transcript elongation of rpl5 occurred (Figure 4B, Additional file 6).

For CW-orf307, the regions that do not include partial orf 288 were used as probes (probes 1-3, Figure 6). We detected a faint band in Taichung 65, the japonica nuclear donor for the CW-CMS line, using probe 1. The detection of this slight signal in Taichung 65 might have occurred via the orf224-like region in probe 1 and the two regions showing more than $85 \%$ identity with orf224 (Figure 5). On the other hand, we detected a CW-mitochondria-specific RNA expression using probes 2 and 3 , suggesting that the downstream regions of $\mathrm{CW}$-orf307 were co-transcribed (Figure 6). Although we checked to see if the presence of the gametophytic restorer gene, $R f 17$ for CW-CMS, would have any effects on the transcript regulation of CW-orf307 in anthers, no differences in transcript patterns were observed between a CMS line and a CW-restorer line, CWR, carrying $R f 17$ and CW-cytoplasm (Figure 6C).

\section{Discussion}

We obtained the entire mitochondrial genome sequences of two rice CMS lines of distinct origin. The master circle of the CW-CMS mitochondrial genome was predicted to contain $559,045 \mathrm{bp}$, whereas that of the LD-CMS genome had 434,735 bp. From a comparison with the recently assembled Nipponbare (japonica), PA64S (japonica), and 93-11 (indica) mitochondrial sequences [9], we found that the CMS genomes were exclusively rearranged (Figure 1). Based on the novel genomic rearrangements and unique ORFs discovered in this study, the mitochondrial haplogroups of the rice cultivars were divided into at least 12 groups (Table 4). It is highly likely that several mitochondrial genomic rearrangements occurred during rice breeding; apparently some rearrangements had deleted the CMS-specific genomic structures (Table 4). For instance, the strongest MCAG candidate of CW-CMS, CW-orf307, was absent from all of the other strains tested (Table 4, Additional file 5). The gene product of CW-orf307 was predicted to be strongly hydrophobic by SOSUI [30], which is consistent with the characteristics of previously known MCAG products [16-19]. Although we failed to detect a transcriptional alteration of CW-orf307 in the presence of $R f 17$, it is still possible that $R f 17$ could be related to translational regulation of CW-orf307. The 


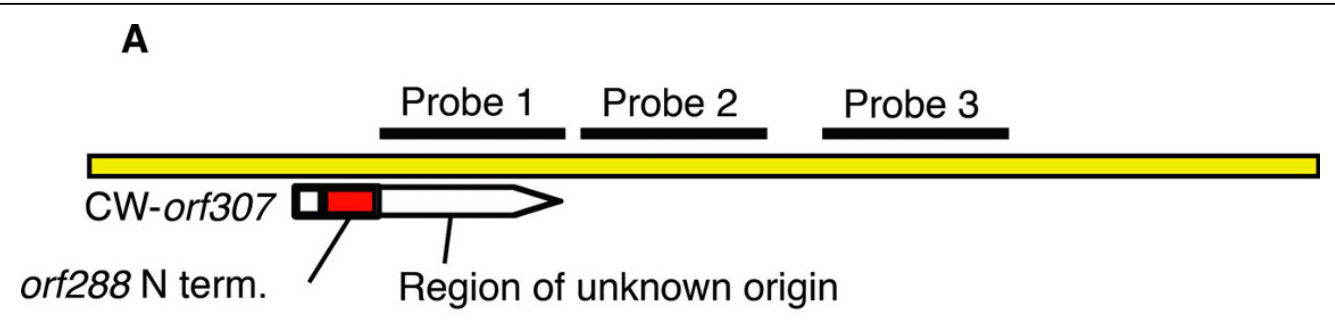

B

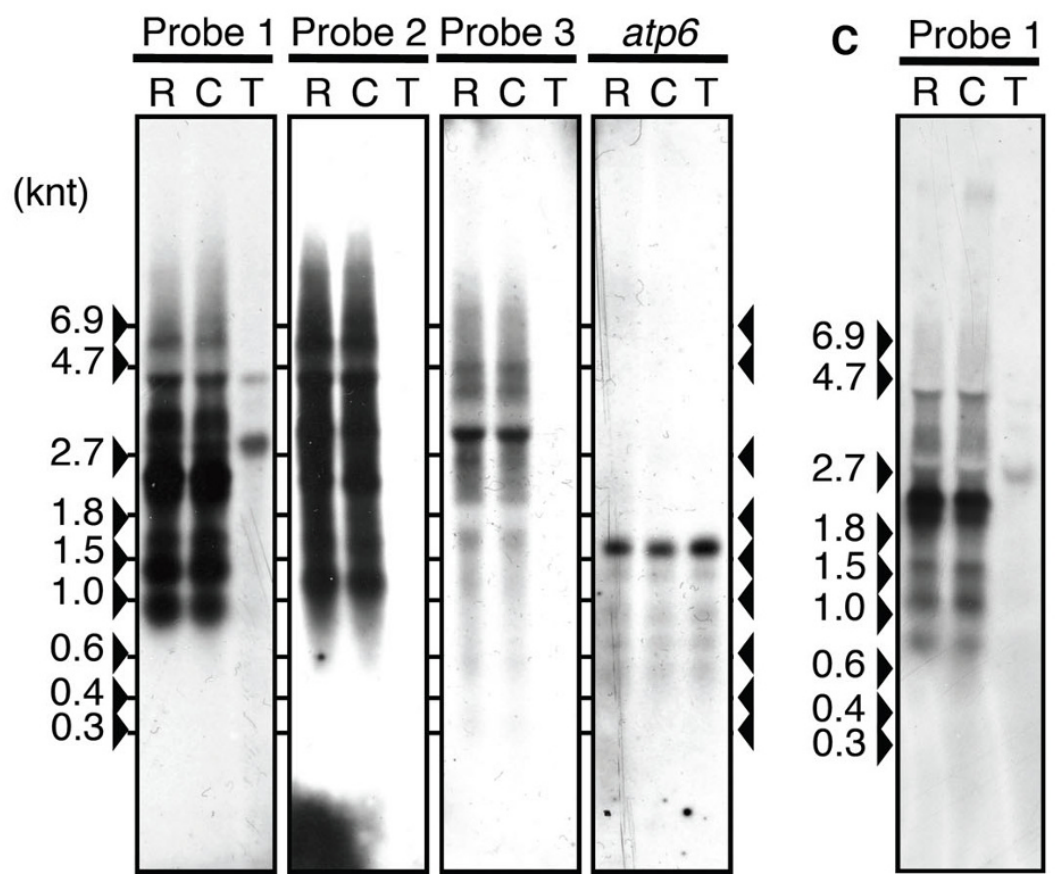

Figure 6 Transcript detection around CW-orf307 locus by northern blot analysis. (A) C-terminus of CW-orf307 and the downstream sequences were probed. (B) Northern blot analysis of mitochondrial RNA extracted from calli using probes 1 to 3. atp6 was used as the internal control. (C) Northern blot using probe 1 to detect CW-orf307 transcript using the total RNA extracted from anthers at the tricellular pollen stage. R, CWR (The restorer line carrying Rf17 and CW cytoplasm); C, CW-CMS line; T, Taichung 65.

presence of orf79 was observed in seven cultivars and in most of the $O$. rufipogon accessions that were previously reported to carry CMS cytoplasm [29], but orf79 was lost from the other cultivars presumably during rice breeding history (Table 4). The reason we speculate that orf79 was "lost" from majority of cultivars is because $O$. rufipogon genomes are generally considered as the ancestors of $O$. sativa cultivars.

The complete copies of orf $152 b$ and orf 288 predicted in the Nipponbare genome were not present in the CWCMS mitochondrial genome. Furthermore, a copy of orf 224 was present in the CW-CMS genome, but its expression was unexpectedly suppressed (Additional file 6), possibly due to alteration of the transcriptional efficiency by the SNP 26 bp upstream of the initiation codon ATG or the two-nucleotide substitutions within the coding region (Table 2). As orf152b and orf288 were expressed in Nipponbare (S.F. and K.T. unpublished data), as was orf224 in this study (Additional file 6), we hypothesize that these ORFs are genes. Therefore, the genetic functions of orf $152 b$, orf 288 , and orf 224 were apparently missing or knocked down in the CW-CMS mitochondrial genome. Among these genes, the introduction of orf 288 in the cultivars seemingly occurred during the rice breeding process. In fact, orf 288 was present in only 35 accessions out of the 69 'core-collection' cultivars and in no accessions from O. rufipogon, indicating that the acquirement of orf 288 at least had definitely occurred during the breeding history (Additional file 5). The absence of orf 288 in approximately half of the cultivars indicates that this gene is obviously not necessary for pollen fertility. Thus the possibility that a loss of function of orf 288 in the CW-CMS line is causing CMS is not likely, because again absence of orf288 apparently does not induce male sterility in such cultivars lacking orf 288 . Whether orf 288 itself can be directly involved in CMS is still an open question, but it may be postulated 
that the presence of orf 288 has contributed to the evolution of a new chimeric CMS gene as a segment of approximately 70 amino acids of $\operatorname{orf} 288$ is identical to $\operatorname{cox} 2$ (data not shown), persuading us that $\operatorname{orf} 288$ is a chimeric gene. Therefore, we conclude that compelling evidence of the 'death' of MCAG via genomic rearrangements during cultivation was demonstrated in this study and, contrarily, that the generation of genes such as orf 288 may be part of the 'birth' process. The 'death' idea is also supported by the fact that the atp6-orf79-like structure was lost from a large proportion of the cultivars (Additional file 5).

The actual mechanism underlying the process of MCAG is considered to be substoichiometric shifting (SSS), which involves the idea that the copy numbers of multipartite mitochondrial genomic molecules (subgenomes) change over generations [31,32]. Interactive recombinations between repeat regions within subgenomes create novel genomic rearrangements, and these novel subgenomes may be present in low copy numbers at first, but they can proliferate and become the majority in subsequent generations by SSS [33]. It has actually been proven that the knockdown of the homologue of the Escherichia coli mismatch repair component in tomato and tobacco unsuppressed the mitochondrial recombination events, and that it eventually caused artificial CMS possibly intermediated by SSS [34]. Therefore, quantitative measurement of the genomic rearrangements we found in the above experiments should provide us with a clearer picture of how the evolution of MCAG took place during rice breeding.

A CMS genome determination study of sugar beet reported a few CMS-specific chimeric structures, other than the best MCAG candidate, preSatp6 [3,18]. Maize CMS sequencing detected one chimeric ORF other than MCAG T-urf13 in CMS-T; none were detected other than the best MCAG candidate orf77 in CMS-S; and none were detected in CMS-C under the 70-amino-acid cut-off criterion [11]. Whether this is meaningful or not, we found 480 ORFs in the Nipponbare genome, 591 in the CW-CMS genome, and 456 in the LD-CMS genome, using the same 70-amino-acid cut-off criterion. Out of the ones that were not present in Nipponbare, none except for the ones we listed (CW-orf307 and LD-orf79) contained a significant portion of the other known genes. Whether any one of these kinds of ORFs is actually related to CMS is still unknown, and it should be emphasized again that Nipponbare as well contains genes that were not described as related to known mitochondrial biological processes but are actually transcribed. The transcribed proportion of these predicted ORFs is undetermined but, based on our current knowledge, detection of any protein products of these genes has not yet been reported [35,36].
On the other hand, the generation and maintenance of a novel chimeric ORF may be allowed by the presence of certain nuclear genes. Possible candidates of such nuclear-encoded genes are pentatricopeptide repeat (PPR)-motif containing genes as discussed below. PPRs are now undoubtedly considered to be involved in posttranscriptional gene regulation events, mostly in plant organelles [37-40]. In higher plant species 400 to 600 PPR genes are encoded, and there is concrete evidence demonstrating that orthologous PPR pairs exist between rice and Arabidopsis [39]. Most of the $R f$ genes (nuclearencoded genes responsible for fertility restoration of CMS) identified to date have been demonstrated to encode a PPR protein [25,41-48]. PPRs encoded by $R f$ genes $(R f-P P R)$ are exceptional because they are not conserved among the species [39]; they are curiously present as heavily duplicated clusters of $R f-P P R$-like genes in the genome. This feature resembles that of diversifying selection acting on the evolution of the disease resistance $R$ genes [49]. There have been a few implications that $R f$ $P P R$ s are actually positively selected $[50,51]$. One perspective involves the expectation that some $R f$ - $P P R$-like genes may be responsible for preventing protein accumulations from certain predicted ORFs that are transcribed but untranslated [40]. There has been no concrete evidence demonstrating that the evolution of $R f-P P R s$ is related to the generation of novel ORFs in mitochondria; however, there certainly must be a selective constraint to the diversifying selection of the $R f-P P R s$, in the same manner as there are pathogens restricting the evolution of the $R$ genes. Our current study suggested that rice mitochondrial genomic rearrangement is an on-going event, and it seems quite reasonable to consider that frequent novel ORF generation as a consequence is the driving force of $R f-P P R$ diversification.

\section{Conclusion}

We have shown that the mitochondrial genomes of the two CMS lines are greatly reorganized in comparison with that of Nipponbare. There appear to be a few genomic structures that are relevant to CMS, and finding these structures within rice cultivars and wild accessions provided us with the idea that genomic recombination events during rice breeding history diminished these unique genomic structures of CMS and, on the other hand, presumably gave rise to novel ORFs.

\section{Methods}

\section{Plant materials}

The origins of two independent rice CMS lines, LDCMS and CW-CMS, were described in our previous studies [22,52]. LD-CMS is derived from the Burmese cultivar Lead rice (Oryza sativa L. ssp. indica) [53], and 
CW-CMS originates from the Chinese wild rice strain W1 (Oryza rufipogon Griff.) [54]. The nuclear donor maintainer line Taichung 65 (japonica) was used as the control for the RNA expression experiment. The development of a restorer line for CW-CMS, CWR, carrying the fertility restorer gene $R f 17$ which functions in a gametophytic manner [52], was described in our previous studies $[52,55]$. The NIAS global rice core collection [27] was obtained from the National Institute of Agrobiological Sciences Genebank (Tsukuba, Japan). O. rufipogon accessions were obtained from the National Institute of Genetics (Mishima, Japan).

\section{Mitochondrial DNA extraction}

Mitochondria were purified on sucrose gradients, as described by Tanaka et al. [56]. The DNeasy plant mini kit (QIAGEN, Hilden, Germany) was used to extract DNA from the mitochondrial fraction.

\section{Sequence assembly}

Pyrosequencing was conducted on the GS-FLX system (Roche, Basel, Switzerland) in TaKaRa-Bio (Otsu, Japan). We obtained 15,965,332-bp nucleotides for the CWCMS genome and 15,020,464-bp nucleotides for the LD-CMS genome. Sequences were assembled to the average of 5,123-bp-long 824 contigs for the CW-CMS genome, and 4,966-bp-long 1,188 contigs for the LDCMS genome, using the GS De Novo Assembler Software (Roche). The average sequence depth, which was defined as the sum of redundancies of each base divided by the length of the respective contig, was 41.74 for the CW-CMS genome and 44.75 for the LD-CMS genome. As many turning points and false linkages were considered, these contigs were manually assembled by the following criteria. (1) The depth of the bridging contigs had to be over 15 , as bridging contigs with a depth under 15 often linked 'dead-end' contigs that were only linked to one another. Also, bridging contigs with a depth under 15 frequently linked fragments that were considered to be nuclear contaminants. (2) 'Dead-end' contigs were ignored. In addition, depth values of these 'dead-end' contigs $(<15)$ were lower than those of nondead-end contigs (>15). (3) Contigs that matched 100\% with the Nipponbare or 93-11 nuclear genomic sequences were ignored as they were considered to be nuclear contaminants and, as in the case of 'dead-end' contigs, depth values of these contigs were always under 15. (4) Plasmid-like sequences, corresponding to B1, B2, $\mathrm{B} 3$, and $\mathrm{B} 4$ reported to be present in rice mitochondria [57,58], were deposited to DDBJ (under accession nos. AB523794 to AB523802), but they were not analyzed in this study. Their characteristics will be presented elsewhere in detail.
These criteria left us with 19 contigs with an average length of $17,877 \mathrm{bp}$ for the CW-CMS genome, and 24 contigs with an average length of 14,828 bp for the LDCMS genome. Following these steps, the candidate linkage of the contigs was validated by PCR analysis (Additional file 7, and see Additional file 8 for the primer sets). Based on the linkage information of the contigs confirmed as well by PCR analysis (depicted in Additional files 9 and 10), master circles were developed for each genome by a 'parsimonious' method, so that each contig appeared at least once to construct the smallest genome. For the $\mathrm{CW}$ mitochondrial genome, there were eight alternative master circle patterns, and 64 patterns for the LD genome. In this paper, one pattern of the master circle was chosen for brevity of presentation (Additional file 11).

The BLASTN program in NCBI tools [59] was used to assign these contigs to the reference Nipponbare mitochondrial genome (DQ167400; described in [9]), and BLASTN was also used to eliminate nuclear genomic contaminations for the above criteria. Handling of the sequences was performed by Sequencher v4.2.2 software (Gene Codes Corporation, Ann Harbor, Minnesota). The Genomematcher v1.2 program [60] was used to plot the sequenced genomes with the reference Nipponbare genome by bl2seq. To avoid the possibility of detecting duplicated regions more than twice, the Nipponbare genome without large duplicated regions $(>10,000 \mathrm{bp})$ synthesized for this study was referenced. Annotated sequences were deposited to DDBJ under accession nos. [DDBJ: AP011076] for CW-CMS and [DDBJ:AP011077] for LD-CMS.

\section{Sequence comparison}

Known mitochondrial genes in Nipponbare were BLASTN searched in the CW-CMS and LD-CMS genomes, and SNPs and Indels were detected. ORFs were attached to the newly sequenced genomes by the est 2 genome program included in the EMBOSS v6.0.0 package [61]. Fifty-six protein-encoding genes (including pseudo-genes), 22 tRNA genes (including pseudo-tRNA) and three rRNA genes analyzed in this study are listed in Additional file 1. To obtain information for the genes that are not present in the Nipponbare genome, gene predictions were performed using the ORF Finder program [59] and Genemark.hmm for the Prokaryotes v2.4 program [62]. Six-frame-translation was performed using the getorf software included in the EMBOSS v6.0.0 package [61]. Since MCAG found in rice BTCMS mitochondria genome encoded a protein of 79 amino acids, the ORFs predicted to encode peptides shorter than 70 amino acids were ignored. 
Information on SNPs and InDels among Nipponbare, PA64S (DQ167807), and 93-11 (DQ167399) was obtained from the study by Tian et al. [9]. We considered it difficult to directly compare the pyrosequence data obtained in this study to that of the sequences obtained by shotgun sequencing in Tian et al. [9] because differences in sequence accuracies between the two strategies might be significant. Thus, instead, known SNPs and InDels in 93-11 vs PA64S [9] were surveyed in LD-CMS and CW-CMS genomes. Phylogenetic analyses of the mitochondrial genomes were performed using RAxML v 7.04 [63].

\section{Construction and screening of the lambda library}

The lambda library was constructed from total DNA isolated from the leaves of CWR, carrying CW cytoplasm, by the CTAB method [64]. DNA partially digested by Sau3AI (TaKaRa-Bio) was ligated into $\lambda$ GEM-11 XhoI half-site arms (Promega, Madison, Wisconsin) using Ligation high (TOYOBO, Osaka, Japan), and packaged using Gigapack III Gold packaging extract (Stratagene, La Jolla, California) according to the manufacturer's protocol. Lambda clones were screened using the probes synthesized from PCR and primer pairs 5'-AGTACCAAAAGCTGCCTCTG-3' and 5'-TTTCCCCCTCATCTTTTAGC-3' for the rpl5 region, and 5'-GCATGATGATCCGATAATC-3' and 5'TAGGTTCGTTATCAGCGG-3' for the CW-orf307 region, respectively. Inserts in isolated clones were subcloned into pBluescript SK- after digestion by BamHI, SacI, SalI, or XbaI (Takara-Bio, Otsu, Japan), and sequenced by the CEQ 8000 Genetic analysis system (Beckman Coulter, Fullerton, California). As a result of sequencing 20,513 bp, two mismatches were detected in comparison with pyrosequencing, thus the accuracy of pyrosequencing was estimated as $99.99 \%$.

\section{Expression analysis}

Mitochondrial RNA was extracted from calli using the typical phenol/chloroform RNA isolation method. For anthers at the tricellular pollen stage, total RNA was extracted using the same method. Mitochondrial RNA $(2.5 \mu \mathrm{g})$ was electrophoresed on $1 \%$ agarose gel after denaturation by formamide treatment and transferred to a nylon membrane. Primers used to design probes for northern hybridization are listed in Additional file 1 .

\section{List of abbreviations}

CMS: cytoplasmic male sterility; InDel: insertion/deletion; MCAG: Mitochondrial cytoplasmic male sterility associated gene; PPR: pentatricopeptide repeat; Rf: restorer of fertility; SNP: single nucleotide polymorphism; SSV: segmental sequence variation.
Additional file 1: Lists of genes and the corresponding primers used for the northern blot analysis. Lists of mitochondrial genes in Nipponbare and the primers used for the northern blot analysis in Figure 6 and Additional file 6.

Additional file 2: Comparison of SNP genotypes among japonica mitochondrial genomes Nipponbare, PA64S, and indica 93-11. Nucleotide positions in 93-11 are displayed. Genotypes in CMS are: j, japonica type; 9, 93-11 type; n, Nipponbare type; p, PA64S type; cms, CMS specific; -, no syntenic region present in the CMS lines. SNPs in the genic regions are given in the last column.

Additional file 3: Comparison of InDel and segmental sequence variation (SSV) genotypes among japonica mitochondrial genomes Nipponbare, PA64S, and indica 93-11. Nucleotide positions in 93-11 are displayed. Genotypes are: j, japonica type; 9, 93-11 type; n,

Nipponbare type; p, PA64S type; $c$, CMS specific. Sequence variations in the genic regions are given in the last column. ${ }^{*}$ Nipponbare vs 93-11 InDels detected in the genomic study by Tian et al. (2006) [9]. Nucleotide positions in 93-11 are indicated. ${ }^{* *}$ Only CW-CMS and LD-CMS genomes retained the same sequences in this region, and other genomes possessed different sequences.

Additional file 4: Phylogenetic relationship of the five rice mitochondrial genomes. All of the sequence variations were used to generate the maximum-likelihood inference-based dendrogram. Bar indicates the rate of nucleotide substitution per site.

Additional file 5: PCR amplification of the CMS unique genomic structure and genes. PCR amplification positive (+) and negative (-) in each rice core-collection accession for each primer pair. * Refer to Figure 2, Additional files 8 and 11 for information on the primers. ${ }^{* *}$ Refer to [28] for details.

Additional file 6: Transcript detection of genes involved in the evolution of the CW-orf307 locus. Transcripts were detected by northern blot analysis using mitochondrial RNA extracted from calli. Genes involved in the rp/5 region (orf165, orf284, cox1, pseudo-rps 14, rpl5, and rps2) and genes involved in CW-orf307 (atp1, orf288, orf224, cox2, and $\operatorname{trnR}$ ). C-terminal region of rps 2 was probed to avoid detecting partial rps2 present in the rp/5 locus. Also, C-terminal orf288, orf224, and cox2 were probed to avoid detecting the fragments in the CW-orf307 transcript. The transcript abundance of atp6 was used as the internal control, and the atp 6 transcript level reflected the ribosomal RNA band intensities detected by ethidium bromide staining. R, CWR (The restorer line carrying Rf17 and CW cytoplasm); C, CW-CMS line; T, Taichung 65.

Additional file 7: Validation of contig linkage by PCR analysis. Lane numbers refer to primer pairs in Additional file 8.

Additional file 8: Information on the primers used for the PCR analysis to validate the linkage between the contigs. Information on the primers used for the PCR analysis in Additional file 7.

Additional file 9: Possible linkage of the contigs validated by PCR analysis for the CW-CMS mitochondrial genome. Numbers within the arrows indicate the contig accession numbers, whereas numbers above indicate the sequence depth of each contig. The yellow color indicates the contigs that appear twice in our master circle, and the blue color indicates the contigs that appear three times. Capital letters represent contig linkages.

Additional file 10: Possible linkages of the contigs validated by PCR analysis for the LD-CMS mitochondrial genome. See the legend for Additional file 9 for descriptions.

Additional file 11: Order and position of the contigs aligned in the master circles. Sequences in this order were deposited to DDBJ (CWCMS, AP011076; LD-CMS, AP011077).

\section{Acknowledgements}

This study was supported by a Grant-in-Aid for Special Research on Priority Areas (No. 18075002) from the Ministry of Education, Science, Sports and Culture, Japan and partly supported by the program for promotion of Basic 
and Applied Researches for Innovations in Bio-oriented Industry (BRAIN). We thank Ms. Yukiko Ikeda for her excellent technical support.

\section{Author details}

'Laboratory of Environmental Biotechnology, Graduate School of Agricultural Science, Tohoku University, 1-1 Tsutumidori-Amamiyamachi, Aoba-ku, Sendai 981-8555, Japan. ${ }^{2}$ Current address: ARC Centre of Excellence in Computer Systems Biology, University of Western Australia, 35 Stirling Highway, Crawley 6009, WA, Australia.

\section{Authors' contributions}

SF designed the study, carried out the experiments, performed sequence analysis, and drafted the manuscript. TK and MY participated in the experiments. KT conceived and supervised the work and edited the manuscript. All authors read and approved the final manuscript.

Received: 14 October 2009 Accepted: 29 March 2010

Published: 29 March 2010

\section{References}

1. McCauley DE, Olson MS: Do recent findings in plant mitochondrial molecular and population genetics have implications for the study of gynodioecy and cytonuclear conflict? Evolution 2008, 62:1013-1025.

2. Kubo T, Newton KJ: Angiosperm mitochondrial genomes and mutations. Mitochondrion 2008, 8:5-14

3. Satoh M, Kubo T, Nishizawa S, Estiati A, Itchoda N, Mikami T: The cytoplasmic male-sterile type and normal type mitochondrial genomes of sugar beet share the same complement of genes of known function but differ in the content of expressed ORFs. Mol Genet Genomics 2004 272:247-256.

4. Unseld M, Marienfeld JR, Brandt P, Brennicke A: The mitochondrial genome of Arabidopsis thaliana contains 57 genes in 366,924 nucleotides. Nat Genet 1997, 15:57-61.

5. Kubo T, Nishizawa S, Sugawara A, Itchoda N, Estiati A, Mikami T: The complete nucleotide sequence of the mitochondrial genome of sugar beet (Beta vulgaris L.) reveals a novel gene for tRNA(Cys)(GCA). Nucleic Acids Res 2000, 28:2571-2576.

6. Handa $\mathrm{H}$ : The complete nucleotide sequence and RNA editing content of the mitochondrial genome of rapeseed (Brassica napus L.): comparative analysis of the mitochondrial genomes of rapeseed and Arabidopsis thaliana. Nucleic Acids Res 2003, 31:5907-5916.

7. Sugiyama $Y$, Watase $Y$, Nagase $M$, Makita $N$, Yagura $S$, Hirai A, Sugiura $M$ The complete nucleotide sequence and multipartite organization of the tobacco mitochondrial genome: comparative analysis of mitochondrial genomes in higher plants. Mol Genet Genomics 2005, 272:603-615.

8. Notsu Y, Masood S, Nishikawa T, Kubo N, Akiduki G, Nakazono M, Hirai A, Kadowaki K: The complete sequence of the rice (Oryza sativa L.) mitochondrial genome: frequent DNA sequence acquisition and loss during the evolution of flowering plants. Mol Genet Genomics 2002, 268:434-445.

9. Tian X, Zheng J, Hu S, Yu J: The rice mitochondrial genomes and their variations. Plant Physiol 2006, 140:401-410.

10. Clifton SW, Minx P, Fauron CM, Gibson M, Allen JO, Sun H, Thompson M, Barbazuk WB, Kanuganti S, Tayloe C, et al: Sequence and comparative analysis of the maize NB mitochondrial genome. Plant Physiol 2004, 136:3486-3503.

11. Allen JO, Fauron CM, Minx P, Roark L, Oddiraju S, Lin GN, Meyer L, Sun H, Kim K, Wang $C$, et al: Comparisons among two fertile and three malesterile mitochondrial genomes of maize. Genetics 2007, 177:1173-1192.

12. Ogihara Y, Yamazaki Y, Murai K, Kanno A, Terachi T, Shiina T, Miyashita N, Nasuda S, Nakamura C, Mori N, et al: Structural dynamics of cereal mitochondrial genomes as revealed by complete nucleotide sequencing of the wheat mitochondrial genome. Nucleic Acids Res 2005, 33:6235-6250.

13. Kubo T, Mikami T: Organization and variation of angiosperm mitochondrial genome. Physiol Plant 2007, 129:6-13.

14. Schnable PS, Wise RP: The molecular basis of cytoplasmic male sterility and fertility restoration. Trends Plant Sci 1998, 3:175-180.

15. Hanson MR, Bentolila S: Interactions of mitochondrial and nuclear genes that affect male gametophyte development. Plant Cell 2004, 16:S154-169.

16. Hack $E_{\text {, Lin }} \mathrm{C}$, Yang $\mathrm{H}$, Horner HT: T-URF 13 Protein from Mitochondria of Texas Male-Sterile Maize (Zea mays L.): Its Purification and
Submitochondrial Localization, and Immunogold Labeling in Anther Tapetum during Microsporogenesis. Plant Physiol 1991, 95:861-870.

17. Nivison HT, Sutton CA, Wilson RK, Hanson MR: Sequencing, processing, and localization of the petunia CMS-associated mitochondrial protein. Plant J 1994, 5:613-623.

18. Yamamoto MP, Kubo T, Mikami T: The $5^{\prime}$-leader sequence of sugar beet mitochondrial atp6 encodes a novel polypeptide that is characteristic of Owen cytoplasmic male sterility. Mol Genet Genomics 2005, 273:342-349.

19. Yamamoto MP, Shinada H, Onodera Y, Komaki C, Mikami T, Kubo T: A male sterility-associated mitochondrial protein in wild beets causes pollen disruption in transgenic plants. Plant J 2008, 54:1027-1036.

20. Kinoshita T: Gene symbols and information on male sterility. Rice Genet News/ 1997, 14:13-22

21. Virmani SS: Heterosis and hybrid rice breeding. Monograph on Theor Appl Genet Springer-Verlag Berlin Heidelberg, Germany 1994, 22:1-189.

22. Itabashi E, Kazama T, Toriyama K: Characterization of cytoplasmic male sterility of rice with Lead Rice cytoplasm in comparison with that with Chinsurah Boro II cytoplasm. Plant Cell Rep 2009, 28:233-239.

23. Iwabuchi M, Kyozuka J, Shimamoto K: Processing followed by complete editing of an altered mitochondrial atp6 RNA restores fertility of cytoplasmic male sterile rice. EMBO J 1993, 12:1437-1446.

24. Akagi H, Sakamoto M, Shinjyo C, Shimada H, Fujimura T: A unique sequence located downstream from the rice mitochondrial atp6 may cause male sterility. Curr Genet 1994, 25:52-58.

25. Wang Z, Zou Y, Li X, Zhang Q, Chen L, Wu H, Su D, Chen Y, Guo J, Luo D, et al: Cytoplasmic male sterility of rice with boro II cytoplasm is caused by a cytotoxic peptide and is restored by two related PPR motif genes via distinct modes of mRNA silencing. Plant Cell 2006, 18:676-687.

26. Kazama T, Nakamura T, Watanabe M, Sugita M, Toriyama K: Suppression mechanism of mitochondrial ORF79 accumulation by Rf1 protein in BTtype cytoplasmic male sterile rice. Plant J 2008, 55:619-628.

27. Kojima Y, Ebana K, Fukuoka S, Nagamine T, Kawase M: Development of an RFLP-based rice diversity research set of germplasm. Breed Sci 2005, 55:431-440.

28. National Institute of Agrobiological Sciences Genebank Global Rice Collection. [http://www.gene.affrc.go.jp/databases-core_collections_wr_en. php].

29. Shinjo $C$, Ishimine $U$, Motomura $K$ : Identification of male strile cytoplasms and fertility restoring genes by seed fertility of $\mathrm{F} 1$ hybrids between male strlie and their restore lines of rice. Jpn J Breed 1982, 32:126, in Japanese.

30. SOSUI. [http://bp.nuap.nagoya-u.ac.jp/sosui/].

31. Small ID, Isaac PG, Leaver CJ: Stoichiometric differences in DNA molecules containing the atpA gene suggest mechanisms for the generation of mitochondrial genome diversity in maize. EMBO J 1987, 6:865-869.

32. Small I, Suffolk R, Leaver CJ: Evolution of plant mitochondrial genomes via substoichiometric intermediates. Cell 1989, 58:69-76.

33. Janska H, Sarria R, Woloszynska M, Arrieta-Montiel M, Mackenzie SA: Stoichiometric shifts in the common bean mitochondrial genome leading to male sterility and spontaneous reversion to fertility. Plant Cell 1998, 10:1163-1180.

34. Sandhu AP, Abdelnoor RV, Mackenzie SA: Transgenic induction of mitochondrial rearrangements for cytoplasmic male sterility in crop plants. Proc Natl Acad Sci USA 2007, 104:1766-1770.

35. Huang S, Taylor NL, Narsai R, Eubel H, Whelan J, Millar AH: Experimental analysis of the rice mitochondrial proteome, its biogenesis, and heterogeneity. Plant Physiol 2009, 149:719-734.

36. Huang S, Taylor NL, Whelan J, Millar AH: Refining the definition of plant mitochondrial presequences through analysis of sorting signals, $\mathrm{N}$ terminal modifications, and cleavage motifs. Plant Physiol 2009, 150:1272-1285

37. Small ID, Peeters N: The PPR motif - a TPR-related motif prevalent in plant organellar proteins. Trends Biochem Sci 2000, 25:46-47.

38. Lurin C, Andres C, Aubourg S, Bellaoui M, Bitton F, Bruyere C, Caboche M, Debast C, Gualberto J, Hoffmann B, et al: Genome-wide analysis of Arabidopsis pentatricopeptide repeat proteins reveals their essential role in organelle biogenesis. Plant Cell 2004, 16:2089-2103.

39. OToole N, Hattori M, Andres C, lida K, Lurin C, Schmitz-Linneweber C, Sugita M, Small I: On the expansion of the pentatricopeptide repeat gene family in plants. Mol Biol Evol 2008, 25:1120-1128. 
40. Schmitz-Linneweber C, Small I: Pentatricopeptide repeat proteins: a socket set for organelle gene expression. Trends Plant Sci 2008, 13:663-670.

41. Bentolila S, Alfonso AA, Hanson MR: A pentatricopeptide repeatcontaining gene restores fertility to cytoplasmic male-sterile plants. Proc Natl Acad Sci USA 2002, 99:10887-10892.

42. Desloire S, Gherbi H, Laloui W, Marhadour S, Clouet V, Cattolico L, Falentin C, Giancola S, Renard M, Budar F, et al: Identification of the fertility restoration locus, Rfo, in radish, as a member of the pentatricopeptide-repeat protein family. EMBO Rep 2003, 4:588-594.

43. Koizuka N, Imai R, Fujimoto H, Hayakawa T, Kimura Y, Kohno-Murase J, Sakai T, Kawasaki S, Imamura J: Genetic characterization of a pentatricopeptide repeat protein gene, orf687, that restores fertility in the cytoplasmic male-sterile Kosena radish. Plant J 2003, 34:407-415.

44. Brown GG, Formanova N, Jin H, Wargachuk R, Dendy C, Patil P, Laforest M, Zhang J, Cheung WY, Landry BS: The radish Rfo restorer gene of Ogura cytoplasmic male sterility encodes a protein with multiple pentatricopeptide repeats. Plant J 2003, 35:262-272.

45. Kazama T, Toriyama K: A pentatricopeptide repeat-containing gene that promotes the processing of aberrant atp6 RNA of cytoplasmic malesterile rice. FEBS Lett 2003, 544:99-102.

46. Komori T, Ohta S, Murai N, Takakura Y, Kuraya Y, Suzuki S, Hiei Y, Imaseki $H_{\text {, }}$ Nitta N: Map-based cloning of a fertility restorer gene, $R f-1$, in rice (Oryza sativa L.). Plant J 2004, 37:315-325.

47. Akagi H, Nakamura A, Yokozeki-Misono Y, Inagaki A, Takahashi H, Mori K Fujimura T: Positional cloning of the rice Rf-1 gene, a restorer of BT-type cytoplasmic male sterility that encodes a mitochondria-targeting PPR protein. Theor Appl Genet 2004, 108:1449-1457.

48. Klein RR, Klein PE, Mullet JE, Minx P, Rooney WL, Schertz KF: Fertility restorer locus Rf1 [corrected] of sorghum (Sorghum bicolor L.) encodes a pentatricopeptide repeat protein not present in the colinear region of rice chromosome 12. Theor Appl Genet 2005, 111:994-1012.

49. Michelmore RW, Meyers BC: Clusters of resistance genes in plants evolve by divergent selection and a birth-and-death process. Genome Res 1998, 8:1113-1130.

50. Geddy R, Brown GG: Genes encoding pentatricopeptide repeat (PPR) proteins are not conserved in location in plant genomes and may be subject to diversifying selection. BMC Genomics 2007, 8:130.

51. Kato H, Tezuka K, Feng YY, Kawamoto T, Takahashi H, Mori K, Akagi H: Structural diversity and evolution of the Rf- 1 locus in the genus Oryza. Heredity 2007, 99:516-524.

52. Fujii S, Toriyama K: Molecular mapping of the fertility restorer gene for ms-CW-type cytoplasmic male sterility of rice. Theor Appl Genet 2005, 111:696-701.

53. Watanabe Y: Establishment of cytoplasmic and genetic male-sterile lines by means of Indica-Japonica cross. Oryza Cuttack 1971, 8(Suppl2):9-16.

54. Katsuo K, Mizushima U: Studies on the cytoplasmic difference among rice varieties, Oryza sativa L. 1. On the fertility of hybrids obtained reciprocally between cultivated and wild varieties. Japan J Breed 1958, 8:1-5.

55. Toriyama K, Hinata K: Anther culture application to breeding of a restorer for a male-sterile cytoplasm of wild rice [ms-CW]. Japan J Breed 1987, 37:469-473.

56. Tanaka N, Fujita M, Handa H, Murayama S, Uemura M, Kawamura $Y$, Mitsui T, Mikami S, Tozawa Y, Yoshinaga T, et al: Proteomics of the rice cell: systematic identification of the protein populations in subcellular compartments. Mol Genet Genomics 2004, 271:566-576.

57. Kanazawa A, Sakamoto W, Nakagahra M, Kadowaki K, Tsutsumi N, Tano S: Distribution and quantitative variation of mitochondrial plasmid-like DNAs in cultivated rice (Oryza sativa L.). Jpn J Genet 1992, 67:309-319.

58. Miyata S, Kanazawa A, Tsutsumi N, Sano Y, Hirai A: Polymorphic distribution and molecular diversification of mitochondrial plasmid-like DNAs in the genus Oryza. Jpn J Genet 1995, 70:601-614.

59. National Center for Biotechnology Information. [http://www.ncbi.nlm.nih. gov/Tools/].

60. Ohtsubo Y, Ikeda-Ohtsubo W, Nagata Y, Tsuda M: A graphical user interface for DNA sequence comparison. BMC Bioinformatics 2008, 9:376.

61. European Molecular Biology Open Software Suite. [http://emboss. sourceforge.net/index.html].

62. Genemark.hmm for the Prokaryotes v2.4. [http://opal.biology.gatech.edu/ GeneMark/gmhmm2_prok.cgi].
63. Stamatakis A: RAxML-VI-HPC: maximum likelihood-based phylogenetic analyses with thousands of taxa and mixed models. Bioinfomatics 2008, 22:2688.

64. Murray MG, Thompson WF: Rapid isolation of high molecular weight plant DNA. Nucleic Acids Res 1980, 8:4321-4325.

\section{doi:10.1186/1471-2164-11-209}

Cite this article as: Fujii et al:: Discovery of global genomic reorganization based on comparison of two newly sequenced rice mitochondrial genomes with cytoplasmic male sterility-related genes. BMC Genomics 2010 11:209.

\section{Submit your next manuscript to BioMed Central and take full advantage of:}

- Convenient online submission

- Thorough peer review

- No space constraints or color figure charges

- Immediate publication on acceptance

- Inclusion in PubMed, CAS, Scopus and Google Scholar

- Research which is freely available for redistribution 